\title{
O efeito das enzimas hialuronidase e tripsina na liquefação do sêmen de macacos pregos (Cebus apella)
}

Regina Celia Rodrigues da PAZ1

Rogério L. ZACARIOTTI ${ }^{1}$ Rodrigo Hidalgo F. TEIXEIRA ${ }^{2}$ Marcelo Alcindo de Barros Vaz GUIMARÃES ${ }^{1}$

\section{Correspondência para:} REGINA CÉLIA RODRIGUES DA PAZ Rua Domingos Russo, 120-Jardim Sandra 18031-210 - Sorocaba/SP

repaz@usp.br

Recebido para publicação: 12/05/2004 Aprovado para publicação: 21/09/2005

1 - Departamento de Reprodução Animal da Faculdade de Medicina Veterinária e Zootecnia da Universidade de São Paulo, São Paulo - SP 2 - Fundação Parque Zoológico de São Paulo, São Paulo - SP

\section{Resumo}

Os efeitos das enzimas hialuronidase e tripsina foram avaliados quanto à liquefação, motilidade, vigor e integridade do acrossoma, no sêmen de seis macacos pregos (Cebus apella), mantidos na Fundação Parque Zoológico de São Paulo. O sêmen foi colhido por eletroejaculação, após anestesia geral, e a fração líquida foi imediatamente avaliada. A fração coagulada do sêmen foi tratada com as enzimas hialuronidase e tripsina, na dose de $1 \mathrm{mg} / \mathrm{ml}$, diluídas em meio 199 (Nutricel, Campinas/SP, Brasil), na proporção 1:4 e examinada após 5 e 15 minutos. Test t de Student foi utilizado para comparar os tratamentos. Não houve diferença significativa quanto a motilidade, vigor e integridade de acrossoma ( $\mathrm{p}>0.05)$, entre a fração de sêmen coagulado diluído em hialuronidase e tripsina, após cinco ou quinze minutos. No entanto, houve diferença significativa quanto a motilidade e vigor entre a fração líquida e a coagulada do sêmen $(p<0.05)$, após quinze minutos. Não houve diferença significativa com relação à integridade de acrossoma ( $p>0.05)$ entre a fração líquida e coagulada do sêmen, após 15 minutos. De acordo com os resultados, podemos concluir que não houve efeito aparente na fração coagulada do sêmen tratado com as enzimas hialuronidase e tripsina com relação a motilidade, vigor e integridade do acrossoma. No entanto, houve diferença significativa entre a fração líquida e coagulada do sêmen com relação a motilidade e vigor, porém não quanto à integridade do acrossoma. De maneira geral, em ambos os tratamentos, não houve a completa dissolução do coágulo.

\section{Introdução}

O sêmen da maioria dos primatas torna-se viscoso após a exposição ao ar, tornando-se um material gelatinoso de difícil acesso para determinação de suas características, que normalmente são avaliadas sob microscopia de luz, após confecção de lâmina coberta por lamínula ${ }^{1}$.

Enzimas proteolíticas como a tripsina e a colagenase foram utilizadas no processo de liquefação de sêmen de primatas ${ }^{2,3,4,5,6,7,8,9,10,11}$, porquinho da índia ${ }^{12}$ e alpacas ${ }^{13}$. Da mesma maneira, enzimas como a colagenase, a fibrinolisina, a hialuronidase e a tripsina foram utilizadas na dissolução do sêmen de lhamas e $\operatorname{alpacas}^{14}$.

No entanto, embora alguns autores recomendem a dissolução da fração coagulada do sêmen de primatas com enzimas proteolíticas, por afirmar que grande quantidade de espermatozóides encontra-se retido no coágulo $3,4,5,6,8,9,10$, outros afirmam que a dissolução química interfere na qualidade do sêmen obtido, além do fato de afirmarem que apenas pequena quantidade de espermatozóides fica retida no coágulo, podendo portanto ser desprezada ${ }^{15,16,17,18}$.

Diante das disparidades encontradas 
na literatura, este estudo torna-se importante pelo fato de avaliar os efeitos de duas enzimas (hialuronidase e tripsina) na motilidade, no vigor e na integridade do acrossoma de espermatozóides da fração coagulada do sêmen. Espermatozóides encontrados na fração líquida do sêmen também foram avaliados quanto a motilidade, o vigor e a integridade de acrossoma.

O objetivo principal deste estudo foi determinar os efeitos da hialuronidase e da tripsina na liquefação, na motilidade e no vigor do sêmen de macacos pregos, após 5 e 15 min.; e na integridade do acrossoma, após 15 minutos da diluição, comparando os resultados encontrados com os resultados obtidos da análise imediata da fração líquida do sêmen.

\section{Materiais e Métodos}

\section{Animais}

Foram utilizados seis exemplares de macaco prego (Cebus apella), adultos, machos, de procedência indeterminada $\mathrm{e}$ fenótipos semelhantes. Os animais foram mantidos na Fundação Parque Zoológico de São Paulo.

\section{Eletroejaculação}

Os animais foram previamente anestesiados com auxílio de caixa de prensa, utilizando-se a associação TiletaminaZolazepan $\left(\right.$ Zoletil $\left.^{\circledR}\right)$ na dose única de $7 \mathrm{mg} /$ $\mathrm{kg}$, via intramuscular.

A eletroejaculação foi realizada utilizando-se aparelho para colheita de sêmen de bovinos domésticos, com controle de voltagem de 0 a 12 volts e eletrodo com $9 \mathrm{~mm}$ de diâmetro e $11 \mathrm{~cm}$ de comprimento, contendo tiras longitudinais em cobre ${ }^{9,19}$.

\section{Avaliação do Sêmen}

Imediatamente após a ejaculação foram avaliadas as características físicas do ejaculado, iniciando-se pelo volume da fração líquida e medição de $\mathrm{pH}$. Uma gota desta fração foi depositada em lâmina coberta por lamínula e avaliada em Microscópio de Luz.
Os espermatozóides foram avaliados quanto a motilidade (0-100\%) e vigor (0-5). Uma alíquota desta fração foi diluída em formol salina $10 \%$ para avaliação da concentração espermática e das características morfológicas do sêmen (\%). A concentração espermática (x $10^{6} / \mathrm{ml}$ ) foi realizada com auxílio de câmara de newbauer e as características morfológicas foram avaliadas em câmara úmida, pela contagem de 100 células/lâmina em Microscópio de Contraste de Fase ${ }^{20}$. Um total de $3 \mathrm{ml}$ de sêmen foi depositado em lâmina de vidro, juntamente com $3 \mathrm{ml}$ de corante para confecção do esfregaço, onde posteriormente 100 células/lâmina foram avaliadas em Microscópio de Contraste de Fase quanto à integridade do acrossoma ${ }^{21}$.

As enzimas hialuronidase e tripsina foram diluídas em meio 199 (Nutricel, Campinas/SP) na concentração $1 \mathrm{mg} / \mathrm{ml} \mathrm{e}$ adicionadas na proproção de $25 \%$ do volume do ejaculado em placas de petri contendo as frações coaguladas e mantidos em mesa aquecedora a $37^{\circ} \mathrm{C}$ pelo período de 15 minutos $^{14}$. Motilidade e vigor foram avaliados após 5 e 15 minutos da adição das enzimas. Da mesma maneira a integridade do acrossoma dos espermatozóides foi avaliada, como descrito acima, após quinze minutos da adição das enzimas ao sêmen coagulado.

\section{Análise Estatística}

O Test t de Student foi utilizado para avaliar as diferenças entre a qualidade do sêmen da fração líquida e da fração coagulada após cinco e quinze minutos da adição das enzimas e entre as frações coaguladas de sêmen diluídas em hialuronidase e tripsina após cinco e quinze minutos ${ }^{22}$.

\section{Resultados}

A tabela 1 mostra a M/EPM dos ejaculados quanto ao volume, motilidade, concentração, anormalidades e integridade de acrossoma da fração líquida do sêmen. A tabela 2 mostra a M/EPM da motilidade, 
vigor e integridade do acrossoma do sêmen coagulado diluído em hialuronidase e tripsina após quinze minutos. As figuras 1 a 3 mostram o volume, a motilidade e a integridade do acrossoma do sêmen coagulado, diluído em hialuronidase e tripsina, individualmente para cada animal.

Não houve diferença significativa quanto a motilidade e vigor ( $p>0.05)$, entre a fração de sêmen coagulado diluído em hialuronidase e tripsina, após cinco ou quinze minutos. Também não houve diferença significativa, entre a integridade de acrossoma dos espermatozóides encontrados na fração líquida e na fração coagulada, diluída em hialuronidase ou tripsina $(\mathrm{p}>$ $0.05)$, após 15 minutos. No entanto, houve diferença significativa quanto a motilidade e vigor da fração líquida, comparada à fração coagulada do sêmen, diluída em hialuronidase $(p=0.009 ; p=0.008)$ e tripsina $(p=0.02 ; p$ $=0.03)$, após quinze minutos. De maneira geral, tanto a hialuronidase como a tripsina foram parcialmente eficientes na dissolução do coágulo, sendo que nenhuma das duas enzimas utilizadas o dissolveram totalmente.

Tabela 1 - Volume, motilidade, vigor, pH, concentração, anormalidades e integridade de acrossoma deespermatozóides da fração líquida do sêmen de macacos pregos, São Paulo, 2003

\begin{tabular}{lcccccccc}
\hline Animais & $\begin{array}{c}\text { Vol. } \\
(\mathrm{ml})\end{array}$ & $\begin{array}{c}\text { Motil. } \\
(\%)\end{array}$ & $\begin{array}{c}\text { Vigor } \\
(1-5)\end{array}$ & $\mathrm{pH}$ & $\begin{array}{c}\text { Conc. } \\
\left(\times 10^{6}\right)\end{array}$ & $\begin{array}{c}\text { Anormais } \\
(\%)\end{array}$ & $\begin{array}{c}\text { Normais } \\
(\%)\end{array}$ & $\begin{array}{c}\text { Int. } \\
\text { Acros. } \\
(\%)\end{array}$ \\
\hline $\begin{array}{c}\text { Macaco } \\
\quad\end{array}$ & 0.4 & 90 & 5 & 8 & 1.0 & 57 & 43 & 55 \\
$\begin{array}{c}\text { Macaco } \\
2\end{array}$ & 0.7 & 90 & 5 & 9 & 5.8 & 36 & 64 & 97 \\
$\begin{array}{c}\text { Macaco } \\
\quad 3\end{array}$ & 0.9 & 60 & 3 & 8 & 1.9 & 23 & 77 & 86 \\
$\begin{array}{c}\text { Macaco } \\
4\end{array}$ & 0.7 & 70 & 4 & 8 & 0.1 & -- & -- & 90 \\
$\begin{array}{c}\text { Macaco } \\
5\end{array}$ & 1.0 & 80 & 2 & 7 & 7.8 & 33 & 67 & 9 \\
$\begin{array}{c}\text { Macaco } \\
6\end{array}$ & 0.4 & 40 & 2 & 7 & 0.1 & -- & -- & -- \\
M/EPM & $0.7 \pm 0.1$ & $71.7 \pm 7.9$ & $3.5 \pm 0.6$ & $7.8 \pm 0.3$ & $2.8 \pm 1.3$ & $37.3 \pm 7.2$ & $62.8 \pm 7.2$ & $61.8 \pm 19.7$ \\
\hline
\end{tabular}

- Dados não aferidos devido à quantidade insuficiente de espermatozóides.

Tabela 2 - Motilidade, vigor e integridade do acrossoma de espermatozóides da fração coagulada de sêmen de macacos pregos, após 15 minutos da adição das enzimas hialuronidase e tripsina, São Paulo, 2003

\begin{tabular}{lcccccc}
\hline \multirow{2}{*}{ Animais } & \multicolumn{3}{c}{ Hialuronidase } & \multicolumn{3}{c}{ Tripsina } \\
\cline { 2 - 7 } & $\begin{array}{c}\text { Motilidade } \\
(\%)\end{array}$ & $\begin{array}{c}\text { Vigor } \\
(0-5)\end{array}$ & $\begin{array}{c}\text { Int. Acros. } \\
(\%)\end{array}$ & $\begin{array}{c}\text { Motilidade } \\
(\%)\end{array}$ & $\begin{array}{c}\text { Vigor } \\
(0-5)\end{array}$ & $\begin{array}{c}\text { Int. Acros. } \\
(\%)\end{array}$ \\
\hline Macaco 1 & 30 & 3 & 48 & 20 & 2 & 46 \\
Macaco 2 & 70 & 4 & 59 & 80 & 5 & 59 \\
Macaco 3 & 20 & 1 & 52 & 30 & 2 & 40 \\
Macaco 4 & -- & -- & -- & -- & -- & -- \\
Macaco 5 & 0 & 0 & -- & 0 & 0 & -- \\
Macaco 6 & 0 & 0 & -- & 0 & 0 & - \\
M/EPM & $24.0 \pm 12.9$ & $1.6 \pm 0.8$ & $53.0 \pm 3.2$ & $26.0 \pm 14.7$ & $1.8 \pm 0.9$ & $48.0 \pm 5.6$ \\
\hline
\end{tabular}

- Dados não aferidos devido à quantidade insuficiente de espermatozóides. 


\section{Discussão}

O sêmen de macacos pregos tornase altamente viscoso após a ejaculação, o que torna sua avaliação bastante prejudicada. Devido a isso, este estudo foi realizado com o intuito de demonstrar o efeito das enzimas hialuronidase e tripsina na liquefação, motilidade, vigor e integridade de acrossoma de sêmen de macacos pregos mantidos em cativeiro.

Para dissolução do coágulo as enzimas hialuronidase e tripsina foram testadas, no entanto, ambas mostraram-se apenas parcialmente eficientes na eliminação do coágulo, já que nenhuma das duas permitiu dissolvê-lo totalmente. Isto pode ter ocorrido devido a diluição $(1 \mathrm{mg} / \mathrm{ml})$ ou devido a concentração utilizada $(25 \%$ do volume total de sêmen). Porém, segundo a literatura, essas enzimas foram capazes de diluir sêmen de lhamas e alpacas na mesma diluição e concentração ${ }^{14}$ e segundo Nagle e Denari ${ }^{7}$ tripsina foi eficaz na diluição do coágulo de sêmen de macacos pregos nas concentrações 1 e $2 \%$ a temperatura de 20 a $35^{\circ} \mathrm{C}$, porém em um período superior a 15 minutos.

Não houve diferença entre a hialuronidase e a tripsina com relação à qualidade do sêmen, pois ambas interferiram da mesma maneira, ou seja, diminuindo a motilidade e o vigor espermático. No entanto, a utilização dessas enzimas não determinou alteração significativa relacionada à integridade do acrossoma. Este dado é muito importante, pois determina a funcionalidade do sêmen, ou seja, sua capacidade de penetração no oócito.

A quantidade insuficiente de espermatozóides na fração coagulada do sêmen, após sua diluição, sugere que poucos espermatozóides permanecem nesta fração ${ }^{16,17,18}$.

Em estudos preliminares o método de adição de enzimas mostrou-se adequado, eliminando todo o coagulo de sêmen em primatas $^{5}$. Com relação a motilidade e vigor, também não foram observadas diferenças entre as enzimas utilizadas, após sua adição
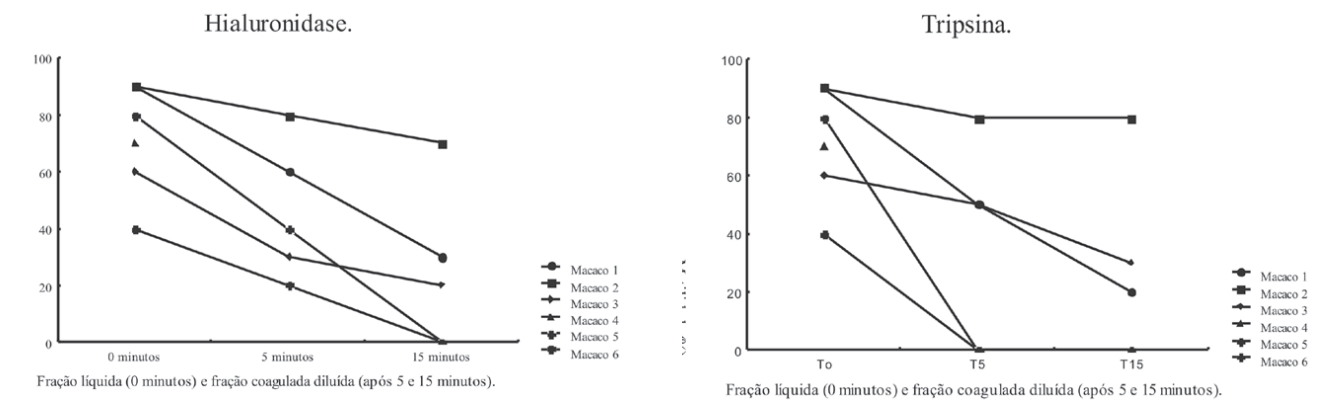

Figura 1 - Efeito das enzimas hialuronidase e tripsina na motilidade da fração coagulada do sêmen de macacos pregos mantidos em cativeiro, São Paulo, 2003
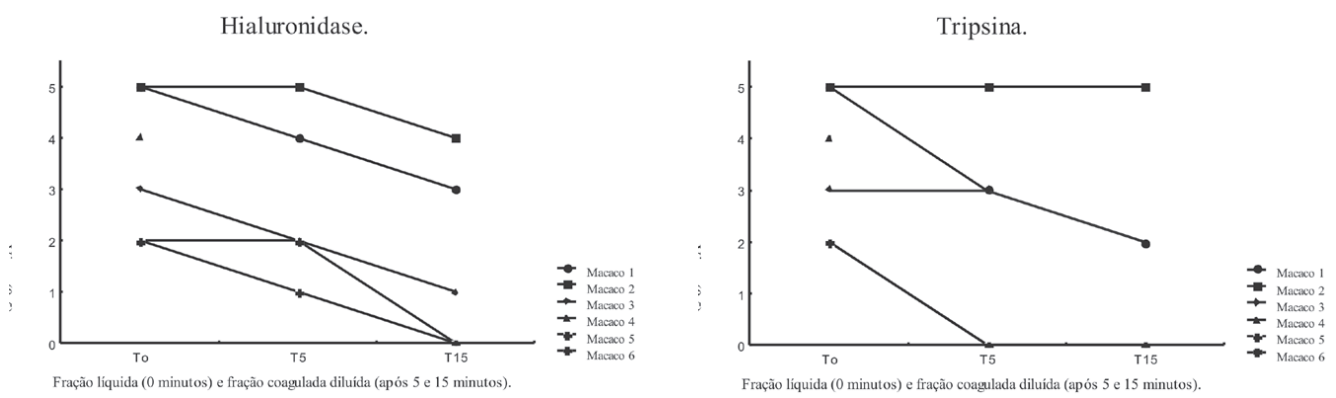

Figura 2 - Efeito das enzimas hialuronidase e tripsina no vigor da fração coagulada do sêmen de macacos pregos mantidos em cativeiro, São Paulo, 2003 

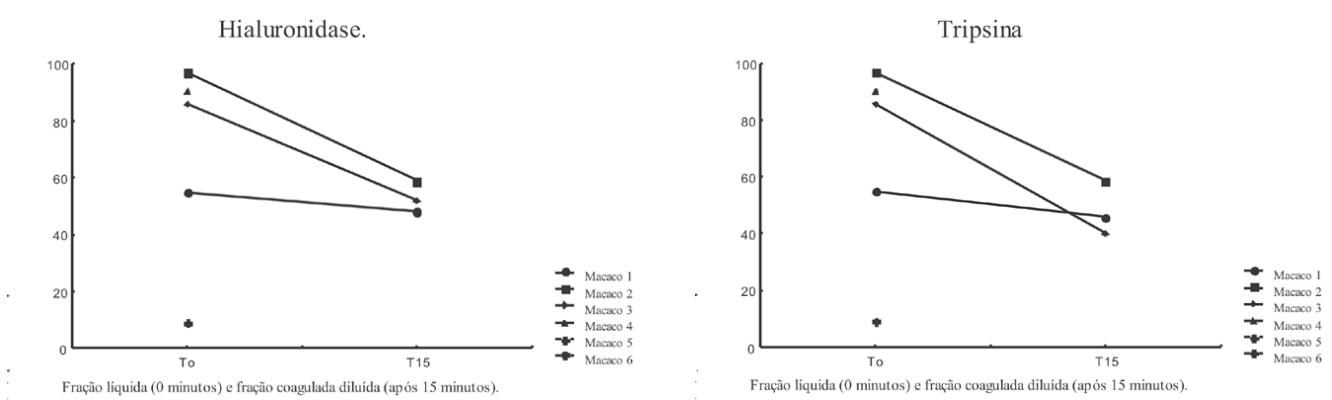

Figura 3 - Efeito das enzimas hialuronidase e tripsina na integridade de acrossoma da fração coagulada do sêmen de macacos pregos mantidos em cativeiro, São Paulo, 2003

ao coagulo de sêmen, em trabalhos anteriores ${ }^{13,12,3,8}$. Em estudo recente realizado por Bravo, Ccallo e Garnica ${ }^{14}$, não houve alteração na integridade do acrossoma relacionada à enzima ou ao tempo de exposição à mesma.

\section{Conclusão}

As enzimas testadas neste experimento não foram efetivas na eliminação completa do coágulo de sêmen de macacos pregos no período máximo de 15 minutos.
Tanto a hialuronidase quanto a tripsina promoveram a diminuição da motilidade e do vigor espermático de acordo com o tempo de exposição, quando comparada a fração líquida do sêmen. Não houve aumento na porcentagem de acrossomas lesados após 15 minutos de exposição às enzimas.

\section{Agradecimentos}

Fundação Parque Zoológico de São Paulo/SP.

\section{The effect of hyaluronidase and trypsin enzymes on liquefaction of semen in capuchin monkey (Cebus apella)}

\section{Abstract}

The effect of the enzymes hyaluronidase and trypsin were recorded on the motility, vigor and acrosome integrity in the semen of capuchin monkey (Cebus apella). The animals $(n=6)$ were maintained at Fundação Parque Zoológico de São Paulo. Under anesthesia semen samples were collected by electroejaculation. Immediately after the ejaculation, the semen liquid fraction was analyzed for volume $(\mathrm{ml})$, $\mathrm{pH}$, motility (\%), vigor (0-5), concentration (cells/ml), defects (\%) and percentage of intact acrosome $(\%)$. The coagulated fraction was treated with a solution of hyaluronidase or trypsin, $1 \mathrm{mg} / \mathrm{ml}$ in commercial medium (199-Nutricel, Campinas/SP, Brazil) in a proportion of 1:4 and the samples were examined after 5 and 15 minutes. The Student T Test (95\%) was used to compare the treatments. There was no significant difference in the motility, vigor or acrossome integrity $(p>0.05)$ between coagulated fraction diluted either in trypsin or in hyaluronidase, after 5 or 15 minutes. However, there was significant difference in motility and vigor between liquid and coagulated fraction, after 15 minutes, for both treatments $(p<0.05)$, but there were no difference in acrosome integrity $(p>0.05)$. In conclusion, there were no apparent effects in the coagulum for both treatments regarding motility, vigor and acrosome integrity. There were significant differences between liquid and coagulated fractions
Key-words:

Cebus apella. Reproduction. Semen. Hyaluronidase. Trypsin. 
regarding motility and vigor, but not for acrosome integrity. In both enzyme treatments there were no complete dissolution of the coagulum.

\section{Referencias}

1 VALE, R. D. R. Características físicas e morfológicas do sêmen de Alouatta caraya (Humboldt, 1812) mantidos em cativeiro, 2002. Dissertação (Mestrado em Reprodução Animal), Faculdade de Medicina Veterinária e Zootecnia, Universidade de São Paulo, São Paulo.

2 BUSH, D. E.; RUSSEL, L. H.; FLOWERS, A. I.; SORENSEN, A. M. Semen evaluation in capuchin monkey (Cebus apella). Laboratory Animal Science, v.25, p.588-593, 1975 .

3 GREER, W. E.; ROUSSEL, J. D.; AUSTIN, C. R. Prevention of coagulation in monkey semen by surgery. Journal of Reproduction and Fertility, v. 15, p. 153155, 1968.

4 HENDRICKX, A. G.; KRAMER, D. C. Reproduction and breeding tecnhiques for laboratory animals: Primates. Philadelphia: Les \& Febigen, 1970. p. 316335.

5 HOSKINS, D.; PATTERSON, D. Prevention of coagulum formation and recovery of motile spermatozoa from Rhesus monkey semen. Journal of Reproduction and Fertility, v. 13, p. 337-340, 1967.

6 KRAMER, D. C.; VERA CRUZ, N. C. Collection, gross characteristics and freezing of baboon semen. Journal of Reproduction and Fertility, v. 20, p. 345348, 1969.

7 NAGLE, C. A.; DENARI, J. H. The Cebus apella monkey (Cebus apella). In: HEARN, JOHN (Ed.) Reproduction in new world primates - New models in medical science. Edimburg: MTP Press Limited 1982. p.39-67.

8 ROUSSEL, J. D.; AUSTIN, C. R. Enzymic liquefaction of primate semen. Journal Fertility, v. 3, p. 288-290, 1967.

9 ROUSSEL, J. D.; AUSTIN, C. R. Improved electroejaculation of primates, Journal of he Institute of Animal Technicians, v. 19, n. 1, p. 22-32, 1968.

10 SCHAFFER, N.; CRANFIELD, M.; MEEHAN, T.; KEMPSKE, S. Semen collection and analysis in the conservation of endangered nonhuman primates. Zoo Biology, p. 47-60, 1989. Supplement 1.

11 SRIVASTAVA, P. N.; FAROQUI, A. A.; GOULD, K.G., Studies of hydrolytic enzymes of chimpanzee semen. Biology of Reproduction, v. 25, p. 363-369, 1981.

12 FREUND, M. Collection and liquefaction of guineapig semen. Proc. Soc. Exp. Biol. Med, v. 98, p. 538540, 1958.
13 BRAVO, P. W.; PACHECO, C.; VILCAPAZA, V. QUISPE, G.; ORDONEZ, C. Degelification of alpaca semen and effect of dilution rates on artificial insemination outcome. Archieves Andrology, v.43, p.239-246, 1999.

14 BRAVO, P. W.; CCALLO, M.; GARNICA, J. The effect of enzymes on semen viscosity in Ilamas and alpacas. Small Ruminant Research, v. 38, p. 91-95, 2000.

15 BARNABE, R. C.; GUIMARÃES, M. A. B. V.; OLIVEIRA, C.A.; BARNABE, A. H. Análise de alguns parâmetros normais do espermograma de macaco-prego. (Cebus apella Linnaeus, 1758). Brazilian Journal Veterinarian Animals Science, v.39, n.6, p.331-333, 2002.

16 REZNICHEK, R. C.; ROUSSEL, J. D.; MANGELSON, N. L.; KADO, B. S.; COCKETT, A. T. $\mathrm{K}$. Some morphologic and biochemical observations of semen in nemestrina monkeys destined for space flight. Fertility \& Sterility, v. 19, n. 3, p. 376-381, 1968.

17 VALERIO, D. A.; LEVERAGE, L. E.; MUSTER, J. H. Semen Evaluation in macaques. Laboratory Animal Care, v. 20, p. 734-740, 1970.

18 VAN PELT, L. F.; KEYSER, P. E. Observations on semen collection and quality in macaques. Laboratory Animal Care, v. 20, n. 4, p. 726-733, 1970.

19 GUIMARÃES, A. B. V. Contribuição para o estudo da colheita e avaliação do sêmen do macaco prego Cebus apella (Erxleben, 1777). 1994. Dissertação (Mestrado em Reprodução Animal) - Faculdade de Medicina Veterinária e Zootecnia, Universidade de São Paulo, São Paulo.

20 BLOM, E. On the evaluation of bull semen with special reference to the employment for artificial insemination, Thesis, Conpenhage. 1950.

21 POPE, C. E.; ZHANG, Y. Z.; DRESSER, B. L. A simple staining method for evaluating acrossomal status of cat spermatozoa. Journal of Zoo and Wildlife Medicine, v. 22, n. 1, p. 87-95, 1991.

22 STATISTIC ANALYSES PROGRAM. $992^{\text {th }}$ ed. STAT SOFT INC. 